\title{
La carrière politique de René Chaloult : l'art de promouvoir une politique nationaliste tout en sauvegardant son avenir politique
}

\section{Denis Chouinard et Richard Jones}

Volume 39, numéro 1, été 1985

URI : https://id.erudit.org/iderudit/304326ar

DOI : https://doi.org/10.7202/304326ar

Aller au sommaire du numéro

Éditeur(s)

Institut d'histoire de l'Amérique française

ISSN

0035-2357 (imprimé)

1492-1383 (numérique)

Découvrir la revue

Citer cet article

Chouinard, D. \& Jones, R. (1985). La carrière politique de René Chaloult : l'art de promouvoir une politique nationaliste tout en sauvegardant son avenir politique. Revue d'histoire de l'Amérique française, 39(1), 25-50.

https://doi.org/10.7202/304326ar d'utilisation que vous pouvez consulter en ligne. 


\title{
LA CARRIÈRE POLITIQUE DE RENÉ CHALOULT: L'ART DE PROMOUVOIR UNE POLITIQUE NATIONALISTE TOUT EN SAUVEGARDANT SON AVENIR POLITIQUE
}

\author{
DENIS CHOUINARD \\ RICHARD JONES \\ Département d' histoire \\ Université Laval
}

La publication, au cours des années 1960 et 1970, d'une pléthore de travaux sur le nationalisme a pu laisser croire que les Québécois ont été obnubilés par ce phénomène, que leurs comportements ont été fortement influencés par les discours nationalistes. Certains historiens, cependant, sans doute un peu en réaction à cette vague, ont laissé entendre que son impact sur la population restait souvent bien problématique $^{1}$.

La carrière politique de René Chaloult ${ }^{2}$, député à l'Assemblée législative du Québec de 1936 à 1952, revêt un intérêt particulier pour celui qui étudie le nationalisme canadien-français et qui cherche à évaluer son importance dans la société. Faut-il attribuer ses succès électoraux à la popularité des thèses nationalistes qu'il mettait inlassablement de l'avant? Sans vouloir écarter complètement cette explication, nous croyons que des nuances s'imposent: de fait, le flair politique de cet esprit indépendant, conjugué à un contexte dont il savait tirer profit, a contribué grandement aux quatre victoires électorales de Chaloult. De même, lorsque la conjoncture se modifia durant les années 1950 et que le Québec, sur le plan politique, s'installa dans la stabilité duplessiste, les possibilités qu'avait Chaloult de se comporter en indépendant disparurent pratiquement. Le député fut emporté en 1952 dans un contexte moins favorable et une tentative de retour en 1956 se solda aussi par un échec. De surcroît, les problèmes personnels qui devaient le miner au cours des dernières années de sa vie mais qui l'affligeaient déjà, ont pu nuire, au dire de ses proches, à sa performance.

\footnotetext{
I Voir Yves Roby, Les Québécois et les investissements américains (1918-1929) (Québec, Les Presses de l'Université Laval, 1976), 117; Susan Mann Trofimenkoff (éd.), Abbé Groulx, Variations on a Nationalist Theme (Toronto, Copp Clark Publishing, 1973), 12 et Action française: French Canadian Nationalism in the Twenties (1975): 117; Jacques Rouillard, Histoire de la CSN (1921-1981) (s.1., CSN et Éditions du Boréal Express, 1981), 47.

2 René Chaloult a tracé certains points forts de sa carrière dans ses Mémoires politiques (Montréal, Éditions du Jour, 1969). Le lecteur pourra aussi consulter la thèse de maîtrise de Donald Thomas, La carrière politique de René Chaloult de 1936 à 1952, Université de Montréal, 1980 , où il trouvera beaucoup d'informations sur les activités de Chaloult en Chambre. Thomas n'a malheureusement pas dépouillé l'important Fonds Paul-Gouin et il s'est très peu servi du Fonds René-Chaloult qui n'avait pas encore été déposé aux Archives nationales du Québec.
} 
La carrière fort mouvementée de Chaloult jette aussi de précieux éclaircissements sur les dissensions internes qui ont conduit plusieurs des groupements nationalistes au cimetière des espoirs déçus. En effet, autant le camp nationaliste ne cessait de déplorer les comportements du «pauvre petit peuple» qui, généralement, ne se ralliait pas autour de lui dans un élan d'unité, autant il était lui-même victime de désaccords et de déchirements profonds dans ses propres rangs. Ainsi, les malheurs qui ont ravagé l'Action libérale nationale et qui ont contribué à détruire le Bloc populaire nous instruisent grandement sur les misères récentes du Parti québécois. D'une part, ces déboires s'expliquent en grande partie par les conflits de personnalité, fréquents dans cette armée de généraux qu'a été la troupe nationaliste. D'autre part, les chefs nationalistes, forts de leurs convictions, croyaient souvent détenir le monopole de la vérité et tendaient à dédaigner les compromis qui, pour eux, ressemblaient davantage à des compromissions. Chaloult, par contre, fit montre d'une certaine souplesse, beaucoup plus en tout cas qu'un Hamel, un Gouin ou même un Groulx. Par ailleurs, il sut habituellement adapter son nationalisme aux besoins du moment. Toutefois, lui aussi avait des principes à défendre qui eurent tôt fait de l'éloigner de toutes les formations politiques qu'il a côtoyées.

A vrai dire, si Chaloult rentrait aisément dans un parti, il ne lui répugnait nullement d'en sortir aussi facilement si son orientation ne lui plaisait pas. Selon ses propres mots, il cherchait «une étiquette interchangeable» ${ }^{3}$. D'une élection à l'autre, son comportement demeurait imprévisible. A des commettants qui cherchaient à savoir, à la veille de l'élection de 1944, sous quelle étiquette leur député allait se présenter, Chaloult répondit le plus sérieusement du monde: «Je ne suis toujours pas fixé là-dessus! ${ }^{4}$

Au cours de sa carrière, donc, René Chaloult fut amené à collaborer tantôt avec les «bleus», tantôt avec les «rouges». Mais il adhéra aussi à l'Action libérale nationale, au Parti national et au Bloc populaire, et, dans les années 1960, se joignit au Regroupement national du Dr René Jutras. A certains moments il entretint même des relations cordiales avec les créditistes. N'avons-nous pas ici l'exemple par excellence du vire-capot en politique québécoise, à côté de qui les Armand Lavergne, Israël Tarte, Oscar Drouin et Rodrigue Biron paraissent bien ternes? Voici, en effet, celui que Daniel Johnson surnommait, en 1956, la «Mae West du Canada français», avec ses «neuf divorces depuis $1930 ! »^{5}$

\footnotetext{
3 Archives publiques du Canada, Fonds Paul-Gouin (ci-après FPG), vol. 81, discours s.d. (circa début 1944).

4 Ibid.

5 Montréal-Matin, 5 juin 1956, 2.
} 
De manière générale, le vire-capot, celui qui n'a pas d'allégeance fixe, qui rejette un parti et se joint à un autre, a mauvaise presse. Alors que de larges fractions de l'électorat appuient toujours un même parti politique et se targuent de leur loyauté indéfectible, - et cela était encore plus vrai à l'époque de Chaloult, - le vire-capot est dépeint comme infidèle, une «girouette» qui a du mal à se «brancher» et qui, évidemment, place en premier lieu ses intérêts personnels. Certes, Chaloult avait une tout autre interprétation de son comportement et il ambitionnait de se classer au rang du petit nombre d'apôtres, hommes indépendants vibrant de foi et d'amour pour leur race, qui mettraient les idées avant les partis ${ }^{6}$. Mais des observateurs comme Gérard Filion qui osait entonner un hymne «à la gloire des vire-capot» ${ }^{7}$, étaient décidément rares!Sauf en 1936, donc, Chaloult ne donna jamais son appui inconditionnel au parti qu'il favorisait afin, disait-il, de se garder les coudées franches. Mais dans le système britannique basé sur des partis disciplinés, celui qui ne s'attache à aucune formation a alors les dés pipés contre lui. La perte du patronage et une maigre caisse électorale peuvent suffire à écourter une carrière politique déjà rendue difficile par les combats en Chambre, où le franc-tireur contrarie tout le monde. Mais René Chaloult survécut néanmoins pendant 16 ans.

\section{Le théoricien fait le saut en politique: les élections de 1936}

René Chaloult devient, au cours des années 1920, un disciple de l'abbé Lionel Groulx. Cette filiation l'amène inévitablement à participer au sursaut nationaliste que connaît le Québec au cours de la décennie suivante. Chaloult est ainsi l'un des signataires de la version remaniée du Programme de restauration sociale, d'abord élaboré par les Jésuites. Ce manifeste, qui avance quelques idées nouvelles telles que la nationalisation de l'électricité, deviendra la bible de l'Action libérale nationale, un nouveau parti réformateur dirigé par Paul Gouin auquel Chaloult participera activement.

De la fondation de l'ALN aux élections de 1936, bien des jeux de coulisses ont lieu et la plupart des députés de l'ALN finissent par abandonner Gouin pour se rallier à Duplessis qui fait sien le programme de l'ALN $^{8}$. Chaloult n'a pas été candidat à l'élection de 1935 , trop pris, semble-t-il, par la politique municipale. Au scrutin de 1936, cependant, il est attiré dans l'Union nationale, tout comme Philippe Hamel, et il brigue donc les suffrages dans Kamouraska, «sur l'ordre», dit-il, de Hamel $^{9}$.

\footnotetext{
6 Le Veilleur (pseud. de R. Chaloult), «A travers la vie courante», l'Action française, 18,3 (sept. 1927): 153.

7 Gérard Filion, «A la gloire des vire-capot», Le Devoir, 6 avril 1949, 1.

8 Voir en particulier Patricia G. Reid, Action libérale nationale, 1934-1939, thèse de M.A., Queen's University, 1966 et Patricia Dirks, The Origins of the Union Nationale, thèse de Ph.D., University of Toronto, 1974.

9 Chaloult, «Le Docteur Philippe Hamel,» Oeuvre des Tracts, 407 (mai-juin 1954): 6.
} 
Mais comment Chaloult explique-t-il sa volte-face envers son ancien chef, Paul Gouin? Pour Chaloult, Gouin n'a manifestement pas l'étoffe d'un leader et il lui reproche d'ignorer systématiquement les volontés de ses lieutenants Philippe Hamel, Ernest Grégoire et Oscar Drouin. Chaloult critique Gouin aussi pour son manque d'ardeur lors des enquêtes contre l'ancien régime et il le soupçonne d'être favorable aux «alliances louches» avec l'adversaire libéral ${ }^{10}$. Pour le moment, donc, il range Gouin parmi les traîtres à la cause, avec qui on ne peut pactiser. Grégoire dénonce encore plus vertement son ancien chef, y allant d'une diatribe qui passera à l'histoire sous le nom de «discours crachat» ${ }^{11}$. Paul Gouin n'oubliera jamais cette violente sortie du député de Montmagny et l'événement aura des incidences graves sur ses relations avec le groupe de Québec.

La campagne dans Kamouraska est acerbe. Lors d'une assemblée particulièrement houleuse à Sainte-Anne-de-la-Pocatière, des partisans libéraux tentent de débrancher les fils du haut-parleur durant le discours de Chaloult alors que des militants unionistes souhaitent que Pierre Gagnon, le libéral sortant, parle des «breeches à Vautrin», une allusion aux culottes que le ministre Irénée Vautrin aurait achetées aux frais de l'État pour ses randonnées en forêt. C'est avec une bien mince majorité que Chaloult remporte cette première victoire électorale. Le nationalisme de Chaloult a-t-il joué le moindre rôle dans cette victoire? Ou les électeurs de Kamouraska n'ont-ils pas voté «bleu» tout simplement dans l'espoir d'être du bon bord et parce que le Parti libéral avait été terni par les révélations sensationnelles lors des enquêtes poursuivies avec acharnement par Duplessis au comité des comptes publics ${ }^{12}$ ?

Qu'importe! Chaloult ne semble pas se perdre en conjectures à ce sujet. Il est entré en politique d'abord pour engager la libération économique des Canadiens français ${ }^{13}$. Hamel l'avait convaincu de la nécessité d'abattre la dictature économique, en commençant par le trust de l'électricité. Loin de se considérer le candidat de Duplessis ou de l'Union nationale, Chaloult se targue d'être le défenseur d'une politique, d'une doctrine. En effet, dès cette première expérience électorale, Chaloult affiche son indépendance. Il prétend alors que l'Union nationale est un forum où tous les nationalistes se sont regroupés pour défendre, et éventuellement réaliser des réformes restées jusque-là des théories de cercles fermés. Une semaine après l'élection, il insiste: «Je suis fidèle à $\mathbf{M}$. Duplessis parce que j'ai confiance en lui. (...) Pour

\footnotetext{
${ }^{10}$ Archives nationales du Québec, Fonds René-Chaloult (ci-après FRC), V:178, causerie radiophonique du 22 juin 1936.

1 Reid, op. cit., 120-123.

12 André J. Bélanger et Vincent Lemieux, «Le nationalisme et les partis politiques», Revue d' histoire de l'Amérique française, 22,4 (mars 1969): 555-556.

13 Chaloult, Mémoires..., 65-66.
} 
adhérer à l'Union nationale, nul n'est tenu de renier ses convictions...» ${ }^{14}$

Tristement, pour Chaloult, les doux parfums de la victoire s'évaporeront rapidement. La nouvelle formation de Duplessis se révèle vite plus semblable aux «vieux partis» que le député réformiste a bien voulu le croire, et l'influence des «bleus» y est encore bien présente. Les déceptions ne tardent pas à pleuvoir sur Chaloult. Surtout, l'ami et guide politique de Chaloult, Philippe Hamel, est écarté du cabinet. Chaloult, atterré, proteste vivement auprès de Duplessis. Le nouveau premier ministre menace de priver Chaloult de tout patronage et de ne plus le reconnaître «comme un des siens». Il déclare que s'il se trouvait un Judas parmi les 12 apôtres, il pourrait bien s'en trouver trois ou quatre parmi les 75 nationaux ${ }^{15}$. Pour le député contestataire, la seule solution serait de renverser Duplessis en Chambre et de le remplacer par Hamel; pourtant il ne se fait pas d'illusions à ce sujet. Il songe plutôt à abandonner son siège afin de regagner sa complète liberté de parole et surtout pour ne pas priver ses électeurs «qui, évidemment, sont affamés et auxquels on menace de couper les vivres» ${ }^{16}$.

Alors que le fossé entre Chaloult et Duplessis se creuse, Chaloult, forcément, s'isole. Il avait durement condamné Gouin en l'abandonnant. Il venait de faire campagne contre les libéraux, traitres aux meilleurs intérêts des Canadiens français. Maintenant il voit Duplessis qui s'apprête à trahir, lui aussi. Sa propre carrière parlementaire tire-t-elle à sa fin avant même de commencer? En fait, ce ne sera pas sa dernière élection. Plus jamais, cependant, il ne se présentera candidat officiel d'un parti. En raison de ses démêlés avec Duplessis, sa carrière sera celle d'un indépendant, qui collaborera tantôt avec les uns, tantôt avec les autres, selon ses sympathies et selon les exigences du moment.

\section{A la recherche d'un nouveau parti}

Les théories de l'élite nationaliste, dans lesquelles puise René Chaloult, s'éloignent des préoccupations quotidiennes de la majorité des électeurs québécois en ces temps de crise économique. Comment alors s'étonner de la volte-face d'un politicien comme Duplessis aux lendemains de 1936? Chaloult lui-même affirmera plus tard le caractère idéaliste et élitiste du programme de l'Union nationale en 1936, se demandant même si Duplessis en avait jamais pris connaissance! «Politicien pragmatique, écrira-t-il, il n'y croyait certainement pas. Mais nous, nous y croyions.» ${ }^{17}$

\footnotetext{
14 Le Devoir, 24 août 1936, 3.

15 Centre de recherche Lionel-Groulx, Fonds Lionel-Groulx (ci-après FLG), Chaloult à Groulx, 9 sept. 1936.

16 Ibid.

17 Chaloult, Mémoires..., 66.
} 
Le député nationaliste n'en est pas moins convaincu, à l'époque, de l'apport qu'il a fourni, avec ses amis Hamel et Grégoire, à l'Union nationale pour balayer les libéraux hors du pouvoir. Il croit donc bénéficier d'une certaine emprise sur la population. Toutefois, dans les faits, cette influence du groupe nationaliste ne dépasse guère un cercle relativement restreint. En tout cas, même en reniant Chaloult et les ultranationalistes qui ont si vigoureusement appuyé l'Union nationale et son chef, Duplessis réussit à garder l'appui de la très grande majorité de la députation comme celui de la population qui l'a élu. Amèrement, les nationalistes sont contraints de constater que les grandes théories visant à sauver la race ne valent pas le patronage. Chaloult le découvrira à ses dépens. Plus tard, alors qu'il cherchera à justifier sa position auprès de ses électeurs à Saint-Pascal, les partisans unionistes, triomphants après leurs longues années d'exil, enfin «du côté du patronage», comme l'affirme Rumilly ${ }^{18}$, prendront d'assaut l'estrade et la réunion n'aura pas lieu.

Lors de la session de 1936, Chaloult et les autres dissidents participent au caucus des députés de l'Union nationale bien qu'ils y fassent de plus en plus figure d'indésirables. En effet, ils ne manquent pas une occasion de lancer des avertissements au gouvernement, de pointer les nationalistes d'hier, tel Oscar Drouin, qui persistent à servir le chef unioniste. Rapidement, la situation devient intenable pour Duplessis, qui ne peut endurer très longtemps dans les rangs ministériels des individus qui ne cessent de critiquer ses politiques. En février 1937, donc, Chaloult est expulsé du caucus de l'Union nationale sur les ordres de Duplessis. Devant les courriéristes parlementaires qu'il se rend alors rencontrer, le député dissident qualifie Duplessis de «tyran pire que M. Taschereau, ce qui n'est pas peu dire» ${ }^{19}$. Du même souffle, il leur annonce la tenue prochaine d'une assemblée de protestation. Effectivement, Chaloult, Hamel, Drouin, Ernest Ouellet, Grégoire et Adolphe Marcoux tiennent une manifestation au Manège militaire à Québec. Chaloult y prononce une allocution à l'emporte-pièce dont le thème traduit parfaitement ses sentiments: «Debout! Dehors les usurpateurs!» ${ }^{20}$ Graduellement, un mouvement se dessine qui deviendra un nouveau parti politique, le Parti national. Celui-ci combattra le parti de Duplessis que Chaloult, sarcastique, rebaptise la «Désunion nationale» ${ }^{21}$.

Le Parti national, cependant, vivra le temps d'une rose ${ }^{22}$. Chaloult veut étendre l'organisation à Montréal mais échoue dans sa tentative de

\footnotetext{
18 Robert Rumilly, Histoire de la province de Québec, tome XXXVI: L'autonomie provinciale (Montréal, Fides, 1966), 178

Fonds René-Chaloult (ci-après FRC), IV:116, Le Soleil, 25 fév. 1937.

Le Soleil, ler mars 1937.

La Nation, 1er juil. 1937.

Pour plus de détails sur les hauts et les bas du Parti National, voir Richard Weatherston, A Reformist-Nationalist Political Opposition to Maurice Duplessis, 1936-1939, thèse de M.A., Université d'Ottawa, 1982, 39ss.
} 
convaincre l'avocat Joseph Blain d'en prendre la direction dans la métropole $^{23}$. Par ailleurs, Hamel, qui devra forcément être le principal pilier de ce nouveau parti, est l'objet de critiques fréquentes, même chez les nationalistes. Certains disent de lui que c'est «un toqué, un homme à idée fixe,» confie Groulx à Chaloult ${ }^{24}$. «Il ne veut faire que de l'électricité», écrit Paul Bouchard, du groupe de la Nation, à Gouin ${ }^{25}$. Déjà, en mars 1937, Hamel et ses amis ont essuyé une défaite retentissante dans la Beauce; dans une élection partielle, leur candidat s'est classé troisième, loin derrière le candidat unioniste et celui de l'ALN ${ }^{26}$. Puis, en février 1938, Grégoire mord la poussière aux élections municipales et la honte rejaillit sur le Parti national qui a engagé son prestige dans la lutte.

Toutefois, les relations empreintes de méfiance avec le groupe de Paul Gouin, qui a précédé les gens du Parti national dans la rupture avec Duplessis, s'avèrent le talon d'Achille de cette nouvelle expérience politique. Si Gouin, toujours chef de l'ALN, et Chaloult s'encouragent mutuellement ${ }^{27}$, il apparaît néanmoins que les deux groupes, en dehors de leur opposition commune au régime duplessiste, collectionnent les divergences.

Idéologiquement, les désaccords d'avant 1936, entre Gouin et le groupe de Montréal, d'une part, et Hamel et les nationalistes de Québec, d'autre part, persistent toujours. Gouin se souvient certainement de ses «soldats» qui ont non seulement déserté mais ajouté «la félonie suprême de tirer dans le dos de leur chef» ${ }^{28}$. Hamel est perçu par le groupe de Gouin, et parfois sans doute par Chaloult lui-même, comme intransigeant, imprévisible et plutôt dénué de sens politique ${ }^{29}$. Pensant déjà à sa réélection dans Kamouraska, Chaloult lorgnerait vers le Parti libéral ${ }^{30}$. Autre conflit non négligeable, Gouin ne peut supporter Ernest Grégoire, dont il n'a pas digéré le «discours crachat» de 1936. Pour Gouin, il est inutile de tenter un rapprochement, même préliminaire, tant que Grégoire ne se sera pas excusé publiquement ${ }^{31}$. En outre, du côté de Hamel et de Chaloult, il n'y a aucune raison de supposer que leurs doutes à l'égard de Gouin se soient dissipés.

\footnotetext{
23 FRC, I:3, Chaloult à Joseph Blain, 2 mars 1937 et Blain à Chaloult, 13 mars 1937.

24 FLG, Groulx à Chaloult, 10 janv. 1937.

25 FPG, vol. I, Paul Bouchard à Gouin, 20 oct. 1937

26 Jean Provencher, J.-E. Grégoire: Quatre années de vie politique, thèse de D.E.S., Université Laval, 1969, 138-139.

${ }^{27}$ FPG, vol. IX, Chaloult à Gouin, 27 janv. 1937 et FRC, III:66, Gouin à Chaloult, 25 mai 1937.

28 Gouin, discours du 12 août 1936, cité dans Reid, op. cit., 180.

29 FPG, vol. I, Paul Bouchard à Gouin, 31 mai 1938.

$30 \quad$ FPG, du même au même, 3 juin 1938 .

31 FPG, vol. X, Gouin à Horace Philippon, 7 janv. 1938.
} 
Pourtant, au début de juin 1938, après un discours de Gouin, l'entente entre les deux groupes semble davantage possible. Hamel télégraphie à Gouin ses «chaudes félicitations» et évoque une image saisissante: «Au chevet d'un peuple mourant d'épuisement et de privation, l'entente des bonnes volontés devient un impérieux devoir.» ${ }^{32}$ Malgré des manifestations apparentes de bonne volonté, chaque groupe s'attend à ce que l'autre fasse le premier pas.

Convaincu d'être en position de force ${ }^{33}$, le groupe Gouin décide de tenir un congrès à Sorel en juillet 1938; Chaloult et «ses amis» sont invités à venir collabore ${ }^{34}$ mais ils ont manifestement du mal à décider d'un plan d'action. "Il semble que nos ex-associés procèdent avec la même hésitation qu'à la veille de leur entrée dans l'ALN en 1935, avec les rancoeurs en plus», observe Louis-Philippe Morin, un organisateur de Gouin à Québec. Il croit que l'ALN aurait plus de chances de réaliser ses politiques par une alliance «intelligente» avec des libéraux réformistes, des jeunes surtout, «qu'avec nos rétifs amis» ${ }^{35}$. Gouin rencontre Hamel, Chaloult et Drouin et attribue leur hésitation au refus de Grégoire de lui faire des excuses publiques. Gouin aussi est convaincu que si le groupe de Québec ne se rallie pas, il aura à supporter tout l'odieux de la désunion des forces oppositionnistes ${ }^{36}$. D'ailleurs Gouin et ses lieutenants espèrent que même si Hamel repousse la branche d'olivier, ses partisans déserteront le Parti national pour rallier l'ALN.

Dans ces conditions, l'alliance ne se fait donc pas. Des membres du Parti national se présentent au congrès de Sorel tandis que leurs chefs brillent par leur absence. Chaloult envoie quand même une carte à Gouin. Il se dit empêché par les circonstances de venir à Sorel mais loue le programme de l'ALN et exprime l'espoir «de combattre un jour avec vous ${ }^{37}$. Dans sa réponse, Gouin émet le même voeu ${ }^{38}$. Sporadiquement, au cours des mois suivants, on tend des perches de part et d'autre. Mais tout échoue, même une ultime tentative de former un front commun nationaliste sous le commandement de Maxime Raymond, député fédéral de Beauharnois, devant qui s'effaceraient Gouin et $\mathrm{Hamel}^{39}$. Il semblerait que Raymond n'ait pas manifesté un enthousiasme débordant à la perspective de venir poser comme grand capitaine dans cette galère! Les négociations, tant dans ce sens que dans celui d'un rapprochement ALN-Parti national, traîneront en longueur, pour finalement ne jamais aboutir. Rien n'indique, cependant, que Chaloult doive porter

\footnotetext{
32 FRC, III:66, Hamel à Gouin, 2 juin 1938.

33 FPG, vol. I, Paul Bouchard à Gouin, 3 juin 1938.

34 FPG, vol. II, Gouin à Chaloult, 7 juin 1938 .

35 FPG, vol. IX, Louis-Philippe Morin à Gouin, 12 juin 1938.

36 FPG, vol. X, Gouin à Horace Philippon, 14 juin 1938.

37 FPG, vol. II, Chaloult à Gouin, 25 juil. 1938.

38 FPG, vol. II, Gouin à Chaloult, s.d. (circa fin juil. 1938).

39 Rumilly, Histoire de la province de Québec, tome XXXVII: Premier gouvernement Duplessis (Montréal, Fides, 1968), 243.
} 
la responsabilité de cette suite d'échecs. Au contraire, du groupe de Québec, il semble le plus conciliant.

En avril 1939, par ailleurs, sous prétexte de remanier la carte électorale, Duplessis élimine le comté de Kamouraska. L'Action nationale $\mathrm{y}$ voit une «sale manoeuvre» pour faire disparaitre un adversaire encombrant ${ }^{40}$. Certes, le premier ministre prétend avoir fait une redistribution juste et équitable des comtés, mais il n'en demeure pas moins, comme Chaloult le fait remarquer, que 35 comtés ont moins d'électeurs que Kamouraska. Chaloult jure qu'il restera «dans la vie publique» et «dans cette Chambre» ${ }^{41}$. Il devient donc impérieux pour Chaloult de se trouver un autre fief, mais pour se faire élire, il lui faudra des appuis politiques d'envergure.

\section{Les élections de 1939: un mariage d'amour ou de raison?}

La disparition du comté de Kamouraska incite Chaloult, qui songeait depuis quelque temps à la possibilité de se rapprocher des libéraux, à redoubler d'ardeur dans ses efforts en ce sens. Horace Philippon, proche collaborateur de Gouin à Québec, est au courant des manoeuvres de Chaloult qu'il juge «sensible aux avantages d'un combat avec un groupe qui aurait des cadres, de la finance, des journaux, etc.». Selon lui, Chaloult et Hamel croient que Godbout serait prêt à leur confier des ministères importants ${ }^{42}$. Or, en raison des tractations entre les libéraux et Chaloult, tout espoir de réunir l'opposition nonlibérale devient inconcevable.

Pour Gouin, en effet, le parti libéral de Godbout doit disparaître, car le vrai parti libéral, à ses yeux, est l'Action libérale nationale. Gouin préfère donc tabler sur la formation de l'opposition officielle à Duplessis, le «vieux» parti libéral étant rayé de la carte, que de prendre le pouvoir à l'aide de «la vieille gang libérale, honnie de tous les libéraux bien-pensants». De plus, le chef de l'ALN se dit certain que, de tous les groupes d'opposition, c'est le sien qui a le plus de chances de succès ${ }^{43}$. L'ALN fera donc cavalier seul.

Quant à lui, Chaloult aurait préféré que l'opposition, regroupant les restes du Parti national, l'ALN et le Parti libéral, fasse front commun pour abattre Duplessis. Mais il n'y a pas de doute dans son esprit que, des trois groupes, le Parti libéral apparait le seul véhicule propre à propager ses idées et qu'il serait «impossible» d'en faire autant «dans un tiers parti» ${ }^{44}$.

\footnotetext{
40 FRC, I:5, Roger Duhamel à Chaloult, 3 avril 1939. Son commentaire paraîtra dans l'Action nationale, 13,6 (juin 1939): 516.

$41 \quad$ L'Action catholique, 27 avril 1939, 3.

42 FPG, vol. IX, Horace Philippon à Gouin, 15 mars 1939 et Ibid., 17 mars 1939.

43 FPG, vol. IX, Gouin à Horace Philippon, 16 mars 1939.

44 L'Action catholique, 9 oct. 1939, 15.
} 
De leur côté, en dépit des objections de la «vieille garde» du parti, certains libéraux, avec Godbout et T.-D. Bouchard en tête, pratiquent avec les chefs du Parti national, depuis leur brisure avec Duplessis, la «stratégie de la main tendue ${ }^{45}$. Ils espèrent ainsi gagner le vote des nationalistes qu'ils jugent largement favorable à Duplessis. Or, le comté de Lotbinière, chasse gardée de Joseph-Napoléon Francoeur depuis 1912 jusqu'à sa défaite en 1936, est alors sans candidat libéral, Francoeur étant passé sur la scène fédérale lors d'une élection partielle en 1937. Chaloult s'y présentera donc comme libéral-indépendant et bénéficiera de l'aide des organisateurs libéraux.

Chaloult, en retour, s'engage à mettre sa fougue anti-unioniste au service des libéraux. Toutefois, soucieux de se garder les coudées franches, il refuse de sortir de son comté pour appuyer les candidats de Godbout ${ }^{46}$ et il proclame bien haut sa liberté de pensée en soumettant à ses nouveaux électeurs un programme conforme à ses idées de $1936^{47}$. Mais surtout, le désir de se venger du traître Duplessis transpire autant dans ses discours que dans ceux de Hamel qui l'appuie sans se porter lui-même candidat cette fois-ci ${ }^{48}$.

Si Chaloult acquiert lors de cette campagne électorale l'appui de ses ennemis d'hier, les libéraux, il s'attire par contre les foudres de nombre de ses supporteurs naturels, les nationalistes, dont l'abbé Groulx. En effet, le chef de file nationaliste fait part à Hamel de sa profonde déception face à la décision de Chaloult qui, à son avis, est maintenant «un homme fini» ${ }^{49}$. Inquiété par cette opinion, Hamel rencontre Groulx pour tenter de le convaincre de la bonne foi de Chaloult. Sans ambages, il lui fait remarquer: «Quiconque, dans notre province, accumule contre lui le rouge et le bleu est voué à l'écrasement.» ${ }^{50}$

Rien n'y fait. Doctrinaire et théoricien, Groulx ne peut accepter ces explications empreintes de pragmatisme. Rejetant tout autant, sinon plus, Godbout que Duplessis, il estime qu'il n'est pas nécessaire de faire des pieds et des mains «ni de ruiner politiquement nos dernières réserves d'hommes pour nous donner à choisir entre la typhoïde et la picote ${ }^{51}$. Il va même jusqu'à faire part de son «dégoût» à l'abbé Gra-

\footnotetext{
45 Jean-Guy Genest, Vie et oeuvre d'Adélard Godbout, 1892-1956, tome I, thèse de D. ès L., Université Laval, 1977, 218 et Norman Ward (éd.), The Memoirs of Chubby Power (Toronto, Macmillan, 1966), 348. Power, organisateur libéral au Québec, s'opposera au choix de Chaloult, "one of the most rabid of nationalists.»

46 Rumilly, Histoire de la province de Québec, tome XXXVIII: La guerre de 1939-1945; Ernest Lapointe (Montréal, Fides, 1968), 47.

47 Le Devoir, 27 oct. 1939, 3.

48 Ibid., 12 oct. 1939, 3.

49 FLG, Groulx à Hamel, 9 oct. 1939.

50 FLG, Hamel à Groulx, 12 oct. 1939.

51 FLG, Groulx à Hamel, 13 oct. 1939.
} 
vel, à qui il déclare ne pas comprendre pourquoi Chaloult a réussi à s'entendre avec Godbout, alors qu'il n'a pu le faire avec Gouin ${ }^{52}$.

Pour des raisons que l'abbé Gravel aura du mal à expliquer ${ }^{53}$, la lettre tombe entre les mains de l'Union nationale qui, tentant de montrer en Chaloult un vire-capot qui immole ceux qu'il adulait en 1936 et qui n'est pas loin d'aduler ceux qu'il immolait ${ }^{54}$, s'empresse naturellement de la publier. Voici donc le candidat libéral indépendant durement frappé par son maître spirituel ${ }^{55}$. Chaloult, dépité, soutient à Groulx: «J'ai combattu pour toutes les causes nationalistes depuis plus de 15 ans; je leur ai sacrifié mes intérêts, ceux de ma famille et j'ai risqué ma santé. J'ai reçu des coups, et de durs, de tous les côtés. Il me restait à être frappé par quelques-uns de mes meilleurs amis...» ${ }^{56}$

Malgré ces incidents, Chaloult triomphe de son adversaire dans Lotbinière. Fort de ce résultat, il tente à nouveau de justifier sa position auprès de Groulx. Mais désabusé, il n'y va pas cette fois par quatre chemins: «Seuls quelques fanatiques, sans jugement, et au surplus déloyaux, tels que Pierre Gravel et Paul Bouchard, ne partageaient pas notre sentiment», déclare-t-il. Il prétend à nouveau qu'une alliance avec Paul Gouin, solution idéale en théorie, ne résistait pas à une froide analyse. Gouin, en attaquant à la fois les libéraux et les unionistes, courait tout droit à une défaite qui aurait éclaboussé Chaloult et Hamel au passage et discrédité leurs idées. Par contre, Chaloult pouvait fort bien, du moins temporairement, ne pas combattre le Parti libéral, même s'il le jugeait trop solidaire d'Ottawa. C'était, à son avis, la seule solution «logique et acceptable» ${ }^{57}$.

Par ailleurs, Chaloult n'est pas sans appui. Même le fougueux curé de Notre-Dame-de-Grâce de Québec, l'abbé Edouard-Valmore Lavergne, nationaliste dans l'âme, regrette que les inquiétudes de Groulx

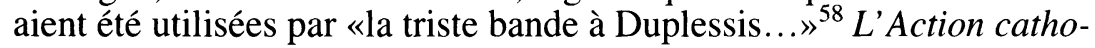
lique aussi, par l'entremise d'Eugène L'Heureux, a appuyé la décision de Chaloult. Qui plus est, l'abbé Groulx, tout en ne partageant pas l'opinion de Chaloult, n'allait pas jusqu'à souhaiter sa défaite; au contraire, il la craignait. Au lendemain de sa victoire, il l'assure qu'il a toujours cru qu'il convient de faire une «différence entre des amis, peutêtre égarés, mais d'esprit loyal, et les professionnels de la trahison» ${ }^{59}$.

\footnotetext{
52 FLG, corr. Groulx-Chaloult, dossier 1928-1939, Groulx à «un ecclésiastique de Québec», 12 oct. 1939.

53 FLG, Pierre Gravel à Groulx, 24 oct. 1939.

54 FRC, III:70, Feuillet de propagande unioniste, s.d. (circa sept. 1939).

55 L'Evénement-Journal, 24 oct. 1939, 3.

56 FRC, III:69, Chaloult à Groulx, 19 oct. 1939.

57 FLG, Chaloult à Groulx, 7 nov. 1939.

58 FRC, III:80, E. V. Lavergne à Groulx, 24 oct. 1939.

59 FLG, Groulx à Chaloult, 26 oct. 1939. Le souligné est de Groulx.
} 
Chaloult, donc, est de retour à la Chambre, tel qu'il l'a promis au moment où Duplessis, triomphant, a éliminé son district électoral. En plus, il peut éprouver une grande satisfaction: «notre Maurice national», comme Hamel se plaît à l'appeler, le traître Duplessis, se voit condamné en 1939 aux banquettes de l'opposition d'où il présidera aux destinées d'une petite phalange d'unionistes. De nouveau, Chaloult est du «bon bord»; d'ailleurs, à la session de 1940, Godbout l'invite au caucus des députés libéraux ${ }^{60}$. Aux unionistes scandalisés de voir des nationalistes appuyer le Parti libéral, René Chaloult réplique que «les plus grands nationalistes du Canada français, Papineau, Lafontaine et Mercier, ont été des libéraux» ${ }^{61}$.

Cette deuxième lune de miel, quoique plus longue que celle de 1936, sera toutefois de courte durée. De fait, n'était-il pas inévitable que les choses se gâtent? En appuyant les libéraux en 1939, Chaloult avait pleinement conscience que sa conduite était dictée par la conjoncture du moment. N'a-t-il pas écrit à Groulx que son alliance avec les libéraux n'était qu'une trêve et ne signifiait nullement une approbation globale de leurs politiques? Principalement, c'était la solidarité du Parti libéral avec Ottawa qui motivait cette prudence du député nationaliste ${ }^{62}$.

Or, dans le contexte de guerre que vit le Canada depuis 1939, les libéraux provinciaux sont appelés à traiter constamment avec Ottawa. Envoyé dans la mêlée, Godbout se targue de défendre, pouce à pouce, l'autonomie du Québec. Toutefois, pour le nationaliste Chaloult, intransigeant devant les propositions fédérales sur les plans financier et législatif, aucune concession n'est justifiable. Il n'est donc pas étonnant que le Parti libéral inquiète bientôt, puis déçoive le député de Lotbinière.

A la Chambre, bien qu'il donne son appui à certains projets de loi du gouvernement, Chaloult prend rapidement figure de trouble-fête pour les ministériels. En juin 1940, au lendemain de l'annonce de l'inscription pour le service militaire au Canada, il présente une motion contre la conscription. Godbout, «imbu de sentiments canadiens», ne peut appuyer la motion Chaloult ${ }^{63}$. Selon le premier ministre, la motion n'est pas justifiée puisqu'il n'est nullement question de conscrire des Canadiens pour service outre-mer ${ }^{64}$.

Déjà ébranlée par les questions relatives à l'effort de guerre, l'alliance Godbout-Chaloult subit encore les assauts des négociations fédérale-provinciales portant sur le rapport Rowell-Sirois paru en mai 1940. Une première rencontre avec Ottawa doit avoir lieu en janvier 1941.

\footnotetext{
60 Chaloult, Mémoires..., 117-118.

61 Rumilly, Histoire..., tome XXXVIII, 108.

62 FLG, Chaloult à Groulx, 7 nov. 1939.

63 Genest, op. cit., tome II: 353.

64 Le Devoir, 19 juin 1940, 3.
} 
Chaloult écrit alors au premier ministre que l'opinion nationaliste se méfie des présumées visées centralisatrices du gouvernement fédéral, qu'elle craint qu'Ottawa ne profite d'une tutelle financière imposée à Québec pour ensuite s'emparer progressivement des compétences provinciales en matière sociale ${ }^{65}$.

Et pourtant Chaloult assure Godbout que son unique désir est «de coopérer loyalement» au bien du Québec, «dans les cadres du Parti libéral» ${ }^{66}$. Dans une lettre au Devoir, il insiste d'ailleurs sur le fait qu'il ne faut pas «blâmer d'avance le Parti libéral provincial en présumant qu'il va céder aux sollicitations d'Ottawa». Prenant comme preuve les promesses de Godbout de ne pas laisser toucher à l'intégrité des droits provinciaux, Chaloult déclare que cette attitude devrait «rassurer les patriotes inquiets» ${ }^{67}$.

Pourtant, on peut penser que Chaloult se range déjà parmi ceuxlà. Dans une lettre qu'il écrit à Godbout au début de 1942, il est clair qu'il prend ses distances face aux libéraux. Il y souligne le fait que le mécontentement des nationalistes à l'égard des libéraux va croissant et rend sa propre situation très délicate. "Comme j'entends rester fidèle à mes principes, je me demande si ma collaboration avec le Parti libéral ne deviendra pas un jour intolérable. ${ }^{68}$ Godbout, cependant, ne répond pas à la lettre ${ }^{69}$; Chaloult lui est déjà perdu.

Ces épisodes marquent la détérioration des relations entre Godbout et le député nationaliste élu sous la bannière libérale en 1939. Tel qu'il l'a lui-même prédit au premier ministre, Chaloult en vient à trouver son alliance avec les libéraux trop gênante. Il quitte donc Godbout. A la session de 1941, Chaloult n'assiste déjà plus au caucus des députés ministériels. En analysant l'attitude du chef libéral, son biographe écrit: «Godbout lui-même devenait plus froid à l'égard de ce député nationaliste qu'il avait fait élire malgré la vive opposition des militants libéraux.»70 La campagne de 1942 contre le plébiscite sur la conscription ainsi que la fondation du Bloc populaire canadien vont sceller l'échec de cette union circonstancielle; pour Chaloult, Godbout va rejoindre Duplessis dans le camp des traîtres aux idéaux des Canadiens français. Godbout ne pardonnera jamais cette volte-face du député indépendant à qui il lancera un jour, en pleine Chambre: «J'ai nourri dans mon sein un serpent. ${ }^{71}$

65 FRC, I:6, Chaloult à Godbout, 6 déc. 1940.

66 Ibid.

67 Le Devoir, 4 janv. 1941, 7.

68 FRC, VI:182, Chaloult à Godbout, 5 janv. 1942.

69 Chaloult a noté sur sa copie de la lettre: «Comme il fallait s'y attendre, cette lettre est demeurée sans réponse.»

70 Genest, op . cit., tome II: 377.

71 Chaloult, Mémoires..., 139. 


\section{Un bloc qui n'en est pas un: Chaloult et le Bloc populaire}

La campagne de la Ligue pour la défense du Canada en vue de mobiliser le «non» lors du plébiscite d'avril 1942 force les nationalistes, ceux de Montréal comme ceux de Québec, ceux oeuvrant sur le plan fédéral comme ceux faisant de la politique provinciale, à oublier pour un temps leurs vieilles rancunes et à collaborer. Pour sa part, Chaloult mène le combat à l'Assemblée législative et participe aussi à des assemblées publiques. Mais sa présence, et surtout celle de Hamel, au sein du comité de la Ligue à Québec, indisposent Edouard Lacroix, industriel de la Beauce, et les deux groupes tiennent leurs assemblées séparément. C'est de mauvais augure.

Le «vote de race» du 27 avril ne clôt toutefois pas la lutte. Au contraire, les nationalistes croient que King n'est pas délié de la promesse qu'il a faite au Québec. Les assemblées de la Ligue se continuent donc. Le 19 mai 1942, au Marché Saint-Jacques, à Montréal, Chaloult $\mathrm{y}$ va d'une diatribe anti-conscriptionniste qui amènera Ottawa à le poursuivre en justice.

Déjà bien connu parce que seul porte-parole de la Ligue en Chambre, Chaloult prend vraiment, avec ce discours et son procès qui fait la manchette des journaux, une stature de figure de proue, de chef en puissance. La défense de sa cause devant les tribunaux devient pour les nationalistes, après le triomphe du «non» au Québec, leur deuxième oeuvre. On amasse des fonds pour aider ce «vaillant défenseur» vers qui «la province entière a les yeux tournés» ${ }^{72}$. Nombreux sont les observateurs qui voient en Chaloult le leader des nationalistes ${ }^{73}$. L'abbé Groulx, pour un, toujours à la recherche du «De Valera» canadienfrançais, lui écrit: «Vous voilà désigné à la foule comme chef.» ${ }^{74}$

Chaloult est acquitté bien que son discours soit condamné ${ }^{75}$. Un banquet est organisé pour fêter la «victoire»: 1500 personnes y assistent. Selon les journaux, de ce banquet surgissent des rumeurs de la fondation d'un nouveau parti. Contrairement à la politique officielle de la Ligue ${ }^{76}$, Chaloult lui-même souhaite depuis longtemps sa transformation en parti politique ${ }^{77}$.

$\mathrm{Si}$, pour Chaloult, le salut de la nation semble exiger ce dénouement, sa propre survivance politique le commande aussi. En effet, durant la campagne du plébiscite, il a, pour ainsi dire, coupé tous les ponts

\footnotetext{
72 Archives de l'Université Laval, Fonds Philippe-Hamel (ci-après FPH), boîte XV, «Lettre circulaire de la Ligue pour la défense du Canada», 21 juil. 1942.

${ }_{73}$ L'Oeil, 15 août 1942; Le Droit, 9 sept. 1942; La Patrie, 13 sept. 1942.

74 FLG, Groulx à Chaloult, 14 août 1942. 105.

La Presse, 3 août 1942 et pour plus de détails sur ce procès, voir Thomas, op.cit., 99-

76 «Communication officielle du mouvement», Le Devoir, 17 juin 1942, 3.

77 FLG, Chaloult à Groulx, 29 avril 1942.
} 
avec Godbout et il n'est pas question pour l'instant d'un rapprochement avec Duplessis. Où donc son fameux pragmatisme l'a-t-il conduit en fin de compte? N'est-il pas maintenant plus isolé que jamais? Où pourrat-il se tourner, sinon vers un nouveau parti, pour être en mesure de promouvoir ses causes sacrées tout en sauvant une carrière politique qui paraît à nouveau compromise? Mieux, dans ce nouveau parti au sein duquel il n'aurait pas à se dire indépendant, il pourrait, servi par son prestige de député et la notoriété acquise lors de son procès, jouer un rôle majeur. La perspective de retourner en Chambre après les prochaines élections accompagné par un bon contingent de nationalistes ne peut que le séduire. Or, la conjoncture politique semble favorable car la question de la conscription et le résultat du plébiscite fournissent aux nationalistes une plate-forme populaire.

De fait, au cours de l'été 1942, René Chaloult est de toutes les tractations visant à former un nouveau parti ${ }^{78}$. Mais trop identifié au docteur Hamel et au Parti national, il n'est pas vraiment pressenti pour en être le chef. D'ailleurs, rien n'indique qu'il convoite le leadership, bien au contraire. On fait donc plutôt appel à Maxime Raymond, politicien prestigieux, ce qui, surtout, permet aux nationalistes de déposer leur pouvoir en terrain neutre. Marie-Louis Beaulieu, un organisateur de la Ligue à Québec qui a travaillé avec acharnement à convaincre Raymond de revêtir le manteau du chef, jubile: «Le passé est effacé..., nous partons en neuf; pas d'Action libérale nationale, pas de Parti national.» ${ }^{79}$

Si Raymond accepte de relever le gant et annonce la formation d'un nouveau parti politique en septembre 1942, tous les nationalistes ne se laissent pas charmer immédiatement. Hamel, en particulier, est un négociateur coriace: il veut profiter de la naissance de ce nouveau mouvement d'envergure pour enfin réaliser la nationalisation du trust de l'électricité. Devant l'impasse, Chaloult, qui a annoncé son adhésion au Bloc lors d'une assemblée publique à Sainte-Croix de Lotbinière, affirme à Groulx qu'il s' «assigne la tâche de travailler de toutes ses forces à l'harmonie et à la bonne entente» en tentant de ramener Hamel à une position moins intransigeante ${ }^{80}$.

En l'absence de Hamel, c'est Chaloult qui anime le mouvement dans la région de Québec, réunissant chez lui des amis «pour causer d'organisation et de projets» ${ }^{81}$. De plus, durant ces semaines de tractations laborieuses et de tergiversations continues, il assume effectivement le rôle de temporisateur. Il rencontre les diverses factions, sert de

\footnotetext{
78 FPG, vol. 1, Marie-Louis Beaulieu à Paul Gouin, 26 juin 1942.

79 Centre de recherche Lionel-Groulx, Fonds Maxime-Raymond (ci-après FMR), Beaulieu à Raymond, 18 août 1942 .

FLG, Chaloult à Groulx, 28 août 1942 et 24 sept. 1942.

81 FMR, Chaloult à Raymond, ler oct. 1942.
} 
pivot aux différents groupes et intervient, à la demande des autres têtes dirigeantes, pour tenter de concilier les positions ${ }^{82}$. Il demeure, cependant, que l'apprenti-médiateur n'est pas le bienvenu partout; par exemple, son passé mine sa crédibilité auprès des unionistes ${ }^{83}$. Il n'a pas, non plus, la confiance des ex-ALNistes à Québec qui reprochent à Hamel et à Chaloult d'avoir «torpillé les mouvements antérieurs» ${ }^{84}$. En outre, toutes sortes de rumeurs visant à salir Chaloult semblent souffler de la Beauce, pays d'Edouard Lacroix, et Gouin, tout comme Chaloult, y voit «une nouvelle manigance de notre excellent ami à tous» ${ }^{85}$. Il apparaît, en effet, que Lacroix voit Chaloult comme principal rival pour la chefferie provinciale du Bloc et cherche donc à le discréditer ${ }^{86}$.

Pendant ce temps, entre Raymond et Hamel, c'est toujours la valsehésitation. Malgré une entente qui intervient finalement entre eux au sujet de l'électricité, la paix ne règne pas pour autant. Sitôt après l'accord, Raymond décide, pour plaire à Lacroix, de tenir la première assemblée à Saint-Georges-de-Beauce et Lacroix ajoute l'insulte à l'injure en précisant qu'il ne tient pas à la présence du duo de Québec. C'est le début de l'imbroglio au cours duquel Chaloult et Hamel, entraînant avec eux Paul Gouin, se révoltent contre les autorités du Bloc ${ }^{87}$. Il semble donc, comme l'écrit Groulx à Chaloult, «que le diable, avec toutes ses puissances, se mette en travers d'entreprises d'où pourrait sortir le salut d'une petite nation chrétienne» ${ }^{88}$. Toutefois, le problème est que pour chacun des intervenants dans cette affaire, Satan n'a pas le même visage!

L'opposition entre Lacroix, d'une part, et Hamel et Chaloult, d'autre part, date de 1935. Hamel et Chaloult ne peuvent supporter cet homme, à leur yeux inculte et sans principe, «un brouillon plein de piastres, un matamore... un gros ignare cossu» ${ }^{89}$. De son côté, Lacroix reproche à Chaloult et à Hamel d'avoir rejoint Duplessis en 1936 et, de plus, d'avoir provoqué la défaite de son candidat lors d'une élection partielle dans la Beauce en $1937^{90}$. En outre, Lacroix est sûr que Chaloult et Hamel, petits bourgeois, sont jaloux de son succès financier, lui qui, à partir de rien, s'est hissé parmi les plus prospères commer-

\footnotetext{
82 FPG, vol. IX, Gouin à Philippon, 3 sept. 1942; vol. II, Chaloult à Gouin, 28 oct. 1942, Gouin à Chaloult, 29 déc. 1942 et Chaloult à Gouin, 2 jan. 1943.

83 FPG, vol. IX, Philippon à Gouin, 8 oct. 1942.

84 Ibid., 15 oct. 1942

85 FPG, vol. II, Gouin à Chaloult, 4 déc. 1942 et FMR, Chaloult à Raymond, 19 nov 1942.

86 Michael Behiels en est convaincu. Voir «The Bloc populaire canadien: Anatomy of Failure, 1942-1947,» Revue d'études canadiennes, 18,4 (hiver 1983-84): 71, note 20.

87 Voir, entre autres, l'article de Behiels ainsi que Paul-André Comeau, Le Bloc populaire, 1942-1948 (Montréal, Québec-Amérique, 1982), 237-251.

88 FLG, Groulx à Chaloult, 30 déc. 1942.

89 FMR, Hamel à Raymond, 10 déc. 1942.

90 FMR, Lacroix à Raymond, 25 janv. 1943.
} 
çants canadiens-français ${ }^{91}$. Il convient donc de remarquer qu'à côté des considérations purement politiques, un conflit de personnalités maintient les deux factions en état de confrontation.

L'enjeu, cependant, est le contrôle de l'organisation de la région de Québec, quelque 35 comtés en tout. D'après son entente avec Raymond, Lacroix a accepté de financer cette région mais, de ce fait, il s'arrogeait le droit d'approuver le programme et le choix de l'organisateur ${ }^{92}$; ainsi, le docteur Pierre Gauthier, lui aussi député fédéral et partisan de Lacroix, sera retenu pour cette fonction.

Pour leur part, Hamel et Chaloult considèrent impensable que l'apport financier repose sur les épaules d'un seul homme, surtout si cet homme s'appelle Edouard Lacroix. En outre, ils craignent que Lacroix, dont les sentiments réformistes ne les rassurent pas, ne devienne chef du Bloc si jamais Raymond démissionnait pour raisons de santé. De toute façon, ils considèrent la région de Québec comme la leur, en raison de leurs états de service, et ils sont convaincus d'être, Hamel avec son prestige et Chaloult avec sa fougue oratoire, ceux que réclament les sympathisants. Dans ces conditions, Chaloult et Hamel, appuyés par Gouin, vont s'abstenir de participer aux activités du Bloc, sauf quelques assemblées qu'ils animent au début du printemps de 1943.

Pour se soustraire à la domination de Lacroix, Chaloult et Hamel proposent de tracer deux zones d'influence dans la région de Québec, l'une pour Lacroix, l'autre pour eux. En juin 1943, Chaloult expose le projet à Georges Pelletier et à l'abbé Groulx, leur demandant d'intervenir auprès de Raymond ${ }^{93}$. Chaloult et Hamel songent aussi aux moyens de trouver des fonds qu'ils pourraient utiliser à leur discrétion et avec lesquels ils pourraient payer leur propre organisateur pressenti, Armand Viau, qui mettrait en branle les souscriptions populaires ${ }^{94}$. Pour trouver les $10000 \$$ nécessaires, Hamel fait notamment appel à son ami Isaïe Beaubien, industriel de Richmond et beau-père de Chaloult ${ }^{95}$.

L'argent recueilli, Raymond refuse pourtant de nommer Viau ${ }^{96}$. Hamel en revient donc au même point: le Bloc doit choisir entre lui et Lacroix. «Il y a une maudite souche dans le chemin», écrit-il à Philippe Girard, organisateur du Bloc à Montréal. «Nous avons le 'bull-dozer'

\footnotetext{
91 FMR, Lacroix à Raymond, 22 nov. 1942 et FRC, V:177, Jean Lacroix à René Chaloult, 7 nov. 1969.

92 FPH, boîte 2, dossier Marie-Louis Beaulieu, 1943-1944, «Procès-verbal du caucus du ler mai 1943». 1943

94 FPG, vol. II, Chaloult à Gouin, 16 déc. 1942.

95 FPH, boîte 3, Hamel à Isaïe Beaubien, ler mars 1943.

96 Raymond prétendra plus tard qu'il ne pouvait le nommer parce que Lacroix n'en voulait pas et aussi parce que certains ecclésiastiques l'avaient prévenu contre Viau, ancien secrétaire d'Athanase David. FMR, Raymond à abbé Médéric Gravel (Port-Alfred, 28 oct. 1943).
} 
pour l'ôter, si monsieur Raymond consent à nous mettre à la besogne.» ${ }^{97}$ Cependant, selon Chaloult, Hamel s'apprête à poser un geste décisif si Lacroix ne part pas. Et, les dés jetés, Chaloult se rangera derrière son ami $^{98}$, d'autant plus que Lacroix multiplie ses attaques contre Chaloult, allant jusqu'à prédire publiquement sa défaite aux prochaines élections ${ }^{99}$. Pour Chaloult, donc, l'heure n'est plus aux compromis.

Curieusement, même s'il menace de démissionner et fait mine de claquer la porte à tout bout de champ, le trio Gouin-Hamel-Chaloult n'est guère pressé de passer aux actes. Pourquoi tant d'hésitations de leur part? Ne soupçonnent-ils pas que, s'ils quittent ou demeurent à l'écart, on s'habituera à se passer de leurs services ${ }^{100}$ ? Ou peut-être ne désespèrent-ils pas non plus de rallier le Bloc à leur position. Quant à Chaloult, il a sans doute une motivation supplémentaire. Le Bloc devait lui permettre de sortir de son isolement politique mais l'imbroglio l'y emprisonne effectivement. Comme son voisin Armand Viau le dit à Raymond: «Notre ami trouve la partie dure. Il est seul et on l'isole terriblement.» 101

Les dissidents pourraient craindre aussi qu'advenant l'effondrement du Bloc, on les accuse d'être les fossoyeurs de ce mouvement que tous les chefs de file nationalistes identifient au plus bel espoir d'unification que les Canadiens français aient vu depuis longtemps. Pour un, Arthur Laurendeau prétend que le public voit la dispute comme une affaire personnelle. Si vous lâchez, dit-il à Chaloult, «toute la rancoeur de la désillusion se ramassera contre vous... On dira qu'il ne s'agit là ou d'un immense orgueil, ou d'un manque de jugement» ${ }^{102}$.

Lorsqu'une élection partielle au fédéral est annoncée dans le comté de Stanstead, Raymond demande au duo de Québec d'y participer ${ }^{103}$. D'autres, jugeant Chaloult plus flexible, croient que le député pourrait s'y rendre seul. Armand Viau essaie même, supposément avec l'assentiment de Chaloult, de convaincre Raymond de choisir Chaloult comme candidat dans Stanstead ${ }^{104}$. Mais Hamel et Chaloult restent sur leurs positions et, avec Gouin, ils parient que leurs talents d'orateurs et leur prestige forceront Raymond à entendre raison. Chaloult, en particulier, insiste sur ses états de service qui devraient lui donner voix au chapitre. N'a-t-il pas été le premier dans la région de Québec à adhérer au Bloc? N'a-t-il pas refusé les «promesses alléchantes» de Godbout? Et, de

\footnotetext{
$97 \quad$ FPH, boîte 13, Hamel à Girard, 8 mai 1943.

98 Centre de recherche Lionel-Groulx, Fonds André-Laurendeau, Chaloult à Laurendeau, 10 mai 1943.

99 FMR, Hamel à Raymond, 3 janv. 1943.

100 FPH, boîte 3, Hamel à Gouin, ler mars 1943.

101 FMR, Armand Viau à Raymond, circa 27 mars 1943

102 FRC, I:8, Arthur Laurendeau à Chaloult, 4 juin 1943.

103 FRC, I:8, Raymond à Chaloult, 16 juin 1943.

104 FMR, Armand Viau à Raymond, 14 juil. 1943.
} 
façon malhabile, il prévient Raymond qu'après l'élection, il entend démasquer Lacroix, «notre second Duplessis» ${ }^{105}$.

La position du trio est cependant fort critiquée. Même leur ami l'abbé Groulx se demande si la démission est «une tactique acceptable quand elle fait surtout l'affaire de l'adversaire». Soutenant que la plupart de ses interlocuteurs appuient Raymond contre le trio, il conseille à Chaloult et à ses amis de rester dans l'espoir de devenir le groupe prépondérant dans le Bloc ${ }^{106}$. A Québec, d'anciens partisans tels MarieLouis Beaulieu, Victor Trépanier et Pierre Letarte trouvent impérieux que les trois mousquetaires rentrent dans les rangs ${ }^{107}$.

Stanstead, qui donne une victoire éclatante au Bloc, affaiblit dangereusement la position de Chaloult et compagnie. En effet, le Bloc semble avoir fait la preuve qu'il pouvait se passer de leurs services. En ce qui concerne Chaloult en particulier, jeune et «plus souple que quelques-uns de ses amis», Groulx croit toujours qu'il sera possible pour le Bloc de le rallier ${ }^{108}$. Il l'encourage donc à reprendre son «rang parmi les chefs de demain» ${ }^{109}$. Armand Viau, qui, il faut le dire, souhaite toujours obtenir le poste d'assistant-organisateur à Québec, prétend que Chaloult endosse la position de Hamel «bien plus par amitié et loyauté que par conviction». Selon lui, il suffirait que Chaloult soit reconnu représentant du Bloc à la Législature pour qu'il se sente apprécié ${ }^{110}$.

Toutefois les relations entre Chaloult et Raymond s'enveniment. Celui-ci est convaincu que Chaloult le perçoit comme un «paravent et un bailleur de fonds» ${ }^{111}$. Raymond, il va de soi, n'est pas heureux non plus d'apprendre de la part de Trépanier que Chaloult croit que lui, Raymond, souffre de «ramollissement du cerveau» ${ }^{112}$. De plus, il accuse Chaloult de faire valoir des exigences irréalistes et lance: «Pour vous, le Bloc, c'est René Chaloult.» ${ }^{13}$ Pour sa part, Chaloult rétorque: «Pour vous, le Bloc, c'est Edouard Lacroix.» ${ }^{114}$ Raymond s'impatiente aussi devant la tendance de Chaloult de parler pour Hamel et Gouin: «Etesvous un Dieu en trois personnes?» lui demande-t-il ${ }^{115}$.

105 FMR, Chaloult à Raymond, 29 juil. 1943

106 FLG, Groulx à Hamel, 23 juin 1943; Groulx à Trépanier, 27 juil. 1943; Groulx à Raymond, 26 juil. 1943.

107 FLG, Trépanier à Groulx, 16 août 1943; FMR, Roland Angers à André Laurendeau, 2 juil. 1943; FRC, I:8, Charles-E. Roy à Chaloult, 8 juil. 1943.

108 FLG, Groulx à Raymond, 17 août 1943

109 FLG, Groulx à Chaloult, 17 août 1943.

110 FMR, Armand Viau à Raymond, 6 et 12 août 1943

111 FMR, corr. Chaloult, «Entrevue de Maxime Raymond avec René Chaloult», 21 août 1943

i12 FMR, corr. Chaloult, note non datée.

113 FMR, Raymond à Chaloult, 29 août 1943.

114 FMR, Chaloult à Raymond, 31 août 1943.

115 FMR, Raymond à Chaloult, non datée, début sept. 1943. 
Les ponts paraissant coupés, Chaloult prépare, selon les mots de Georges Pelletier, «un petit coup de Jarnac» ${ }^{116}$, soit une causerie à la radio où il affirme que la dictature économique est en train d'infiltrer le Bloc ${ }^{117}$. Mais il ménage ses mots, et le public ne semble pas comprendre qu'il vise Lacroix ${ }^{118}$. Même Paul Gouin prétend que «les gens sont sous l'impression que tout est raccommodé au Bloc!» ${ }^{119}$ Il faut donc durcir le ton et lors des causeries subséquentes du trio, les allusions au «trustard» dans les rangs deviendront plus précises. Assailli par des conseils contradictoires venant tant des amis que des ennemis du trio, Raymond finit par enjoindre les dissidents à «rentrer dignement dans le rang s'ils ne veulent pas être considérés comme des adversaires du Bloc» ${ }^{128}$.

Lorsque le Bloc tient son congrès au début de février 1944, les congressistes accordent leur appui à Raymond et n'acceptent pas d'entendre Chaloult, Hamel et Gouin. Les dissidents s'estimeront donc exclus $\mathrm{du}$ parti. Hamel envisage la fondation d'un nouveau mouvement et demande $5000 \$$ au beau-père de Chaloult ${ }^{121}$. Beaubien, qui a promis de l'argent, se ravise maintenant: il ne pourra les aider ${ }^{122}$. Le groupe fait néanmoins une publicité tapageuse à Québec mais Marie-Louis Beaulieu assure Raymond que les partisans du Bloc sont prêts à laisser tomber Hamel et Gouin quoiqu'ils hésitent à abandonner Chaloult ${ }^{123}$.

Pour sa part, le député de Lotbinière se dit découragé et envisage de se retirer de la politique ${ }^{124}$. Comme la Gazette, son ennemi acharné, l'affirme: Chaloult «seems destined permanently for the role of the lone wolf, roaming the cold steppes of ultranationalist isolation without a pack to support him on his forays» ${ }^{125}$. Mais son ami Charles Roy, journaliste à La Presse, l'assure qu'au printemps 1943, quand Raymond était malade, «nous songions tous alors, à l'exception de M. Gouin, à te demander de diriger nos forces dans le champ provincial, si quelque chose d'inattendu et d'imprévisible se produisait» 126 .

En mars, après une nouvelle déclaration de Raymond et une rencontre avortée entre Laurendeau et Chaloult, ce dernier dénonce amèrement le Bloc dans l'enceinte de l'Assemblée législative. La rupture est irrémédiablement consommée et Chaloult doit se poser de sérieuses

116 FMR, Pelletier à Raymond, 31 août 1943.

117 FMR, corr. Chaloult, «Le Bloc populaire canadien au provincial», causerie du 11 sept.

1943.

i18 FMR, Pelletier à Raymond, 13 sept. 1943 et Pierre Letarte à Raymond, 14 sept. 1943.

119 FPH, boîte 11, Gouin à Hamel, 20 sept. 1943.

120 Le Devoir, 30 nov. 1943.

121 FPH, boîte 3, Hamel à Isaïe Beaubien, 9 fév. 1944

122 FPH, boîte 3, Isaïe Beaubien à Hamel, 16 fév. 1944.

123 FMR, Marie-Louis Beaulieu à Raymond, 14 fév. 1944.

124 FPG, vol. 81, Chaloult à Gouin, 16 fév. 1944

125 The Gazette, (Montréal), 16 fév. 1944.

126 FRC, VIII:244, Charles E. Roy à Chaloult, 22 fév. 1944. 
questions sur son avenir politique. En vue de stopper Duplessis, Chaloult semble avoir envisagé, en septembre 1943, la possibilité de demander aux libéraux de financer la lutte du trio contre Duplessis. Choqués, Trépanier et Letarte, à qui il a fait cette confidence à la fin d'une longue nuit passée au chalet de Trépanier, ont rétorqué que les libéraux tiendraient ainsi les nationalistes en otages ${ }^{127}$. Maintenant, au début de 1944, les créditistes semblent avoir l'oeil sur lui, et Réal Caouette lui affirme: «Votre place n'est pas dans le Bloc mais plutôt dans l'Union des Électeurs.»128 De fait, le trio aurait tenté, sans succès cependant, de former une alliance avec le Crédit social ${ }^{129}$. Par ailleurs, la stratégie de Chaloult et Hamel semble déplaire à Gouin qui confie: «Le trio est en train de devenir un duo... Nous restons 'trio' devant le public pour les besoins du moment seulement.» ${ }^{130}$ Gouin ne veut pas d'une nouvelle aventure politique puisque le temps et l'argent manquent. En outre, il semble souhaiter un rapprochement avec le Parti libéral au nom des réformes sociales et économiques qu'il espère voir se concrétiser ${ }^{131}$.

\section{Vers la marginalisation}

En 1944, Chaloult se retrouve dans une situation pour le moins inconfortable. S'étant mis à dos d'abord l'Union nationale, ensuite les libéraux et finalement le Bloc populaire, il est plus isolé que jamais. La défense de ses principes l'a éloigné de toutes les formations; celles-ci, de leur côté, ne sont plus disposées à supporter l'indépendance politique de cet homme imprévisible dont la rentabilité électorale leur apparaît incertaine. Robert Rumilly conseille alors à Chaloult de se rapprocher des indépendants fédéraux, tels Sasseville Roy et Frédéric Dorion, avec qui il pourrait entretenir des liens fructueux ${ }^{132}$. S'il trouve cette formule en principe «excellente», Chaloult craint cependant qu'en pratique ces gens, assez près de Duplessis, soient mal disposés envers lui ${ }^{133}$.

Pour accroître ses chances de réélection, Chaloult décide d'abandonner Lotbinière et de se présenter dans Québec-Comté où l'élément nationaliste dont il dépend paraît plus vigoureux et où il compte beaucoup d'amis. Peut-être aussi considère-t-il que le fait de ne plus disposer du patronage dans Lotbinière lui a aliéné beaucoup d'électeurs.

Chaloult devra affronter les candidats «rouge» et «bleu» et, pour gagner, il lui faudra rallier le vote anti-vieux parti. Le Bloc populaire ne constitue pas un obstacle: malgré sa rupture avec Chaloult, il préfère

127 FMR, corr. Chaloult, «Déclaration assermentée», 9 sept. 1943.

128 FRC, VIII:258, Réal Caouette à Chaloult, 7 fév. 1944.

129 FMR, Marie-Louis Beaulieu à Raymond, 19 avril 1944.

130 FPG, vol. 81, Gouin à Horace Philippon, 27 avril et 8 mai 1944.

131 FPG, Horace Philippon à Gouin, 31 mai 1944.

132 FRC, VII:215, Robert Rumilly à Chaloult, 2 mars 1944.

133 FRC, VII:215, Chaloult à Robert Rumilly, 6 mars 1944. 
bien sûr la victoire de ce dernier à celle de l'unioniste ou du libéral qui pourrait résulter de la division du vote nationaliste. Le Bloc laisse donc le champ libre à Chaloult qui, somme toute, est encore «considéré comme membre de la famille» ${ }^{134}$.

Par contre, les négociations avec le Crédit social se révèlent plus laborieuses. Les militants de l'Union des Électeurs souhaitent présenter leur candidat. Mais J.-E. Grégoire, vice-président du parti, affirme que Chaloult est créditiste «à 95 pour cent» et demande qu'on l'appuie. Chaloult finalement n'aura pas d'adversaire créditiste ${ }^{135}$. Pendant la campagne, il évoque la nécessité d'une «réforme monétaire énergique» pour libérer les Canadiens français de l'«emprise des banques», affirmation qui doit sûrement plaire aux oreilles créditistes.

La lutte est ardue. Cette fois, Chaloult ne bénéficie d'aucun patronage. A la place de bouts de chemin, de ponts et de «jobs», Chaloult promet la lutte contre l'impérialisme britannique et contre la dictature des trusts. Ennemi des deux vieux partis, il les dénonce comme des frères jumeaux «que leur mère elle-même, la dictature économique étrangère, qui les allaite pourtant tous les jours, ne saurait reconnâ̂tre» ${ }^{136}$. Finalement, c'est par une faible marge que Chaloult remporte le comté en se faufilant entre le libéral et l'unioniste.

Réélu, Chaloult veut renforcer ses appuis. Si Raymond échoue dans une ultime tentative de s'entendre avec Hamel et Chaloult ${ }^{137}$, celuici crée des liens étroits en Chambre avec André Laurendeau, un des quatre élus du Bloc. Il rend aussi hommage aux créditistes, ce dont Réal Caouette le remercie ${ }^{138}$. Certes, ces appuis demeurent limités mais Chaloult saura profiter d'une conjoncture politique favorable pour opérer un rapprochement avec Duplessis, celui qu'il n'a cessé de fustiger depuis près d'une décennie. Tranchant avec la politique de collaboration du précédent gouvernement, Duplessis fait de l'autonomie provinciale son cheval de bataille. Sa plaidoirie à l'occasion de la conférence fédérale-provinciale de 1946 amène le député indépendant à écrire chaleureusement au premier ministre: «J'ai fort apprécié... le cran et le panache que vous [y] avez affichés.» ${ }^{139}$ Chaloult s'insurge contre «l'aplaventrisme» passé des «moutons rouges de Québec» devant les «moutons rouges d'Ottawa» et il condamne leurs sympathies présentes pour toutes les influences dissolvantes de la société canadiennefrançaise ${ }^{140}$.

\footnotetext{
134 FRC, VIII:241, Philippe Girard à Chaloult, 26 août 1944

135 FRC, I:9, Chaloult à Emile Benoît, 14 juil. 1944 et Le Devoir, 12 juil. 1944.

136 FPH, boîte 6, «Discours aux électeurs du comté de Québec», fin juil. 1944.

137 FMR, Raymond à Hamel, 25 janvier 1945; FPH, boîte 3, Chaloult à Paul Massé, 25 janv. 1945; FMR, Hamel à Raymond, ler fév. 1945.

138 FRC, VIII:258, Chaloult à Réal Caouette, 27 fév. 1945.

139 FRC, VI:181, Chaloult à Maurice Duplessis, 12 nov. 1946

140 FRC, VI:187, «discours», s. d., 1948.
} 
Dans un contexte où il ne peut guère prendre la vedette dans la défense des grandes causes nationalistes, Chaloult doit pratiquer une politique plus concrète. Ainsi, à la fin de 1947, il admet qu'il n'a peutêtre pas donné à son comté autant de ponts et de routes que les électeurs le souhaitaient mais, chiffres en mains, il s'efforce de démontrer que Québec-Comté a eu plus en octrois et subsides que la plupart des autres circonscriptions $^{141}$.

Les élections de 1948 portent largement sur le thème de l'autonomie provinciale. Chaloult demande aux électeurs de voter pour Duplessis qui «a repris les droits abandonnés par M. Godbout et [qui] nous assure qu'il entend tenir en dépit des promesses et des menaces du gouvernement central» ${ }^{142}$. De plus, il ne peut qu'être reconnaissant envers le chef unioniste qu'il a enfin convaincu d'adopter un drapeau distinctif pour la province. Chaloult voit donc une cause pour laquelle il militait depuis longtemps couronnée de succès. Ce résultat rend encore plus brillante son étoile dans le firmament nationaliste. Chaloult est même perçu comme la «Jeanne d'Arc» québécoise, affirme, extasié, Anatole Vanier, citant l'abbé Groulx ${ }^{143}$ !

Mais comment Chaloult, échaudé en 1936, peut-il encore porter foi aux assurances de Duplessis? Certes, il continue de douter de la profondeur des sentiments nationalistes du premier ministre même s'il se dit impressionné par la vigueur avec laquelle Duplessis a mené les batailles autonomistes de 1944 à 1948. Par ailleurs, il doit être pénible à Chaloult de mettre en veilleuse la lutte contre les trusts et d'oublier les querelles passées. Mais a-t-il le choix? Son avenir politique est maintenant largement entre les mains de Duplessis.

Il ne suffit pas, cependant, que Chaloult fasse l'éloge de l'autonomisme de Duplessis pour que l'Union nationale se jette à ses pieds. Bien des unionistes, dont Paul Bouchard, ne sont guère entichés du député indépendant et ne veulent pas entendre parler d'une alliance avec lui $^{144}$. Mais Rumilly, Houde et d'autres plaident sa cause auprès de Duplessis et les militants unionistes se laissent convaincre d'appuyer Chaloult aux élections de $1948^{145}$.

Bien sûr, tout au long de la campagne, Chaloult clame-t-il son indépendance face à l'Union nationale. Toutefois, malgré des souscriptions totalisant près de $6000 \$^{146}$, il doit recourir à la machine

\footnotetext{
141 FRC, VI: 187, «Discours aux électeurs du comté de Québec», Noël 1947.

142 FRC, V:178, «Discours électoral», 1948.

143 Centre de recherche Lionel-Groulx, Fonds Anatole-Vanier (ci-après FAV), Anatole Vanier à Chaloult, 26 janv. 1948.

144 FRC, VII:215, Robert Rumilly à Chaloult, 4 mars 1948

145 Ibid., 30 juin 1948.

146 FRC, VIII:238, «Bilan financier de l'organisation Chaloult», 1948.
} 
unioniste $^{147}$. Par ailleurs, dans une annonce pleine page parue dans les journaux, il dénonce les libéraux et déclare aux électeurs: «Le choix n'est pas douteux; appuyons l'Union nationale.» ${ }^{148}$

Chaloult est élu facilement cette fois même si, en plus d'un adversaire libéral, il doit affronter un candidat créditiste ${ }^{149}$. Pourtant, suivant une proposition de Rumilly, il a laissé entendre avant les élections que si les créditistes présentaient un candidat dans son comté, il appuierait une candidature nationaliste contre Réal Caouette dans Pontiac, aux prochaines élections fédérales ${ }^{150}$. Mais loin d'être effrayé, Caouette invite Chaloult lui-même à venir se battre contre lui ${ }^{151}$ !

De retour à la Chambre, sa situation est délicate. Seule une poignée de libéraux, surtout anglophones, ont résisté à la marée unioniste. Chaloult, qui a tant vanté l'indépendance, ne peut rentrer carrément dans les rangs unionistes. Au contraire, dans les dossiers controversés de ces années, presque tous d'ordre social et économique, il talonne le gouvernement. Lors des débats sur l'amiantose et pendant la grève de l'amiante, il prend la part des ouvriers contre Duplessis et Barrette, en qui il voit «des avocats zélés des compagnies» ${ }^{152}$. Ce faisant, il s'attire la reconnaissance de Gérard Picard, président de la CTCC ${ }^{153}$, mais ses relations avec Duplessis se détériorent. Et même si Chaloult était tenté de se rapprocher des libéraux - il discute même de programme avec Georges-Emile Lapalme - ${ }^{154}$, aucun accord n'est possible cette fois.

En 1952, Chaloult se trouve donc au pied du mur. Devant l'emprise de l'Union nationale, il doit pouvoir compter sur son appui s'il veut être réélu, d'autant plus qu'il ne réussit pas à convaincre les libéraux de ne pas lui opposer d'adversaire ${ }^{155}$. Par ailleurs, l'opinion nationaliste, que Chaloult se targue de représenter, appuie toujours Duplessis. De plus, ses commettants lui reprochent de ne pas apporter assez de patronage dans le comté, le forçant ainsi à solliciter des montants supplémentaires à la Voirie ${ }^{156}$. De fait, même pour Chaloult, les élections ne se font pas avec des prières. En outre, les souscriptions n'affluent pas cette fois à sa caisse électorale ${ }^{157}$. Chaloult doit donc encore s'aggripper, comme à un mal nécessaire, à la bouée de sauvetage que constitue pour lui l'Union nationale.

\footnotetext{
147 A la fin de la campagne, Chaloult paiera 500\$ à Saül Garneau et $300 \$$ à Emile Boiteau, deux organisateurs unionistes. Chèques retrouvés dans FRC, VIII:235.

${ }_{148}$ Le Devoir, 27 juil. 1948, 7

149 FPH, boîte 5, Chaloult à J.-E. Grégoire, 24 mars 1948

150 FRC, VII:215, Robert Rumilly à Chaloult, 20 fév. 1948

151 FRC, VIII:258, Réal Caouette à Chaloult, 7 avril 1948

152 FRC, I:15-16, Chaloult à Laurendeau, 21 fév. 1949.

153 FRC, I:15-16, Gérard Picard à Chaloult, 11 oct. 1949.

154 FRC, IV:136, Chaloult à Firmin Létourneau, 22 sept. 1951.

155 FRC, VIII:236, Chaloult à André Laurendeau, 30 juin 1952.

156 FRC, VIII:236, Antonio Talbot à Chaloult, 26 juin 1952 et ministère de la Voirie à Chaloult, 7 juil. 1952 .

157 FRC, VIII:237, Emilien Rochette à Chaloult, s.d. 1952.
} 
Malheureusement pour lui, bien des unionistes sont las de ce député qui tantôt défend leurs politiques, tantôt déploie ses ressources oratoires contre elles. Même si l'Union nationale ne lui oppose pas de candidat officiel, un unioniste indépendant se présente, appuyé par une partie de l'organisation locale. Le candidat libéral remporte alors la victoire, profitant de la division du vote unioniste.

La responsabilité de l'Union nationale dans la défaite de Chaloult reste à établir. Certes, Duplessis avait assez peu de raisons de souhaiter le retour de Chaloult à tel point qu'il pouvait même préférer un libéral à cet indépendant embarrassant. Officiellement, pourtant, Duplessis et Jos.-D. Bégin désavouent Larochelle, candidat unioniste indépendant, et expulsent des rangs du parti les organisateurs qui l'avaient appuyé. Rumilly et d'autres essaient de convaincre Chaloult de la bonne foi de Duplessis ${ }^{158}$. D'ailleurs, le chef de cabinet du premier ministre fait savoir à Rumilly que «le patron est assez en maudit contre ces cochonslà qui lui donnent l'apparence d'un homme déloyal» ${ }^{159}$. Mais Chaloult, lui, soupçonne une trahison ${ }^{160}$ que pourrait confirmer l'avenir. En effet, quatre ans plus tard, lorsque Chaloult sera défait de justesse dans Jonquière-Kénogami où il briguait les suffrages comme libéral indépendant, Duplessis aurait exulté ${ }^{161}$.

Les nationalistes pleurent l'échec de 1952 et cachent mal leur amertume devant la «sottise de notre pauvre peuple» ${ }^{162}$, devant ces électeurs ingrats qui auraient considéré que Chaloult ne les avait pas suffisamment «asphaltés». Toutefois, Chaloult tente un retour en 1956 afin de livrer un baroud d'honneur contre les trusts. Cette fois, cependant, celui que les libéraux ont dénoncé en 1948 comme le «commisvoyageur en patriotisme de M. Duplessis» ${ }^{163}$ s'arrête chez eux, charmé par leur nationalisme économique ${ }^{164}$. Son alliance avec le Parti libéral déçoit certains nationalistes qui, s'ils souhaitent la victoire personnelle de Chaloult, favorisent par contre le parti de Duplessis. De fait, pour Chaloult, la conjoncture n'est pas tout à fait mûre pour cette bataille, le Parti libéral non plus d'ailleurs - et lorsque viendront les campagnes de 1960 et 1962, ce sera trop tard pour lui. D'une part, mal à l'aise devant le nationalisme séculier des années 1960 qu'il juge gauchisant ${ }^{165}$ et, d'autre part, accablé par des problèmes de santé, René Chaloult sera disparu de la vie publique au moment où l'équipe du tonnerre réalisera la révolution tranquille.

\footnotetext{
158 FRC, VII:215, Robert Rumilly à Chaloult, 6 juin 1952.

159 FRC, VII:215, Robert Rumilly à Chaloult, 18 juil. 1952.

160 Chaloult, Mémoires...., 162, 288.

161 Pierre Laporte, «Les élections ne se font pas avec des prières», Le Devoir, 4 déc. 1956.

162 FLG, Groulx à Chaloult, 15 août 1952.

163 Le Soleil, 26 juil. 1948, 22.

164 FRC, I:23, Chaloult à Georges-Émile Lapalme, 27 fév. 1956.

165 FAV, Chaloult à Anatole Vanier, 5 sept. 1957.
} 
Il est clair que les positions défendues par Chaloult l'ont amené à préférer le rôle de député d'opposition, même lorsque le parti qu'il appuyait était au pouvoir. En harcelant avec acharnement le gouvernement en maintes occasions, il se retrouvait sur l'avant-scène. Somme toute, il réussit le tour de force de prolonger durant 16 ans une carrière politique comme député indépendant. Mais pour d'abord se faire élire, il dut développer l'art du compromis tout en gardant une certaine inflexibilité en raison de son statut de représentant d'un groupe idéaliste. Sa force fut peut-être de savoir doser ses positions, différencier compromis et compromission, idéal et utopie. C'est ainsi qu'il sut utiliser les partis pour demeurer à la Législature, tribune privilégiée pour la diffusion de son message. Par contre, les partis pouvaient espérer retirer un bénéfice électoral de l'appui de Chaloult, compte tenu de son prestige comme chef de file nationaliste. Certes, l'intérêt de cette collaboration dépendait en grande partie de la conjoncture. Or, la conjoncture changeante de la fin des années 1930 et le contexte particulier de la guerre favorisèrent Chaloult. Mais la domination de l'Union nationale après 1944 et surtout après 1948 ont diminué ses possibilités d'action comme indépendant et entraîné la fin de sa carrière politique.
\end{abstract}

